\title{
ANION-CATION SITE DEPENDENCE OF PRESSURE COEFFICIENTS FOR DONORS IN GaAs
}

\author{
S. BEDNAREK aNd J. ADAMOWSKI \\ Faculty of Physics and Nuclear Techniques \\ Technical University of Mining and Metallurgy (AGH) \\ Al. Mickiewicza 30, 30-059 Kraków, Poland
}

The theoretical model, based on the many-band approach, is proposed for the strongly localized donor states in GaAs. The pressure coefficients for the states of $A_{1}$ and $T_{2}$ symmetry have been calculated for the donors at the anion and cation sites. The obtained results show that these pressure coefficients are different from the conduction-band pressure coefficients and are dependent on the lattice site occupied by the impurity as well as on the symmetry of the donor states.

PACS numbers: 71.55.-i

In GaAs, the substitutional donor impurities of groups IV and VI can form weakly localized hydrogen-like states as well as strongly localized states. Both the types of donor states can yield energy levels in the gap. Under hydrostatic pressure, the hydrogen-like energy levels change according to the associated conduction-band minimum, whereas the energy levels of the strongly localized states are resonant with the conduction band at the ambient pressure and enter the gap at high pressure. The results of experiments [1-4] for the strongly localized donor states in GaAs under hydrostatic pressure have been interpreted in different manners. Liu et al. [1] suggest that all strongly localized donor states possess comparable pressure coefficients (about $1 \mathrm{meV} / \mathrm{kbar}$ with respect to the valence-band maximum). Dmochowski et al. [2-4] argue that the pressure coefficients for the group-IV donors ( $\mathrm{Si}, \mathrm{Ge}, \mathrm{Sn}$ ) take on the values about $2 \mathrm{meV} / \mathrm{kbar}$, but for the group-VI donors $(\mathrm{S}, \mathrm{Se})$ are close to zero. The assignment of symmetry to these states is also not evident. On the one hand, all these states are interpreted as the states of $A_{1}$ symmetry [2-4], on the other hand, the states of $T_{2}$ symmetry cannot be excluded [5]. For the Ge donor, the anticrossing, observed in Ref. [6] under high hydrostatic pressure, between the energy levels of the hydrogen-like $1 s$ state and the strongly localized donor state permits us to assign the $A_{1}$ symmetry to this state.

The pressure dependence of the donor states was the subject of few theoretical papers (e.g. $[5,7,8]$ ). Ilowever, the applied methods are useful either for hydrogen-like donors [5] or for the impurities with deep energy levels $[7,8]$. The donor levels in GaAs, although associated with the donor states of strong localization, are close to the conduction-band minimum. Therefore, we are dealing with the shallow energy levels both for the weakly and strongly localized donor 
states. Such states require a theoretical description, which includes the influence of conduction-band states. The method based on the one-band approximation has allowed us $[9,10]$ to explain the nature of the anomalous anticrossing [6] between the donor energy levels in GaAs. In order to describe the pressure dependence, we have to extend this approach taking into account many conduction bands. In the present work, we discuss the properties of the $A_{1}$ and $T_{2}$ states formed by the donor impurities at the anion and cation sites. The proposed approach takes into account both the symmetry and realistic dispersion relations for several lowest-lying conduction bands.

Let us consider the donor states of the strong electron localization, i.e., the states for which the average electron-donor center separation is of the order of the crystal lattice constant. The formation of such states is mainly caused by the short-range potential arising from the difference between the potentials of the impurity and host crystal atoms. In the present treatment, we neglect the long-range Coulomb potential of the donor center, which contributes to the creation of the weakly localized states with the average electron-donor center distance much larger than the lattice constant. We solve the many-band equation in the wave vector space by the variational method with the trial wave function being the linear combination of the conduction band Bloch functions $\psi_{n} q(r)$. Since we are interested in the energy levels lying in the range of $10 \mathrm{meV}$ below the conduction-band minimum, while the band gap of GaAs is about, $1.5 \mathrm{eV}$, we neglect the influence of the valence bands.

The matrix elements of the short-range potential are proposed in the plane-wave basis in the following form:

$$
V_{k k^{\prime}}=-\sum_{l} \gamma_{l} P_{l}(\cos \theta)=-\gamma_{0}-\gamma_{1} \cos \theta-\ldots
$$

where $P_{l}$ is the Legendre polynomial, $\cos \theta=k \cdot k^{\prime} / k k^{\prime}$, and $\gamma_{l}$ are constant parameters taking on non-negative values. They determine the interaction between the donor center and the electron in the states of the azimuthal quantum number $l$. The parameters $\gamma_{l}$ are assumed to be independent of pressure, which corresponds to the neglect of the influence of pressure on the core electron states. If only $\gamma_{0}$ is different from zero, we obtain the Dirac delta potential in the $r$-space. For sufficiently large $\gamma_{0}$, such interaction generates the localized donor state of $A_{1}$ symmetry ( $s$-like or scalar-like state). On the other hand, if only $\gamma_{1}$ takes on non-zero and large enough values, the localized donor state of $T_{2}$ symmetry is generated ( $p$-like or vector-like state). For the short-range potential of form (1), we get the analytical solutions, which can be written down in the implicit form. We obtain for the $A_{1}$ states

$$
\frac{1}{\gamma_{0}}=\sum_{n \boldsymbol{q}} \frac{\left|\psi_{n} \boldsymbol{q}(0)\right|^{2}}{E_{n} \boldsymbol{q}-E}
$$

and for the $T_{2}$ states

where

$$
\frac{1}{\gamma_{1}}=\sum_{n \boldsymbol{q}} \frac{\left|\boldsymbol{B}_{n} \boldsymbol{q}\right|^{2}}{E_{n} \boldsymbol{q}-E}
$$

$$
B_{n} \boldsymbol{q}=\sum_{\boldsymbol{G}} a_{n} \boldsymbol{q}(\boldsymbol{G}) \frac{\boldsymbol{q}+\boldsymbol{G}}{|\boldsymbol{q}+\boldsymbol{G}|}
$$


and $a_{n} q(G)$ are the Fourier coefficients of the conduction band Bloch functions at the donor center. In order to determine the donor energy $E$ from the above expressions, we have to know the conduction-band Bloch wave functions and dispersion relations $E_{n} q$ in the first Brillouin zone. We have calculated these quantities with the help of the empirical pseudopotential method. The band structure under the hydrostatic pressure has been obtained by introducing the pressure dependent lattice constant and pseudopotential form factors in such a way that we reproduce the band structure known from the experiments. This procedure is in agreement with the general idea of the empirical pseudopotential method. We have taken into account such a number of the conduction bands, which guarantees the convergence of the calculations (usually 6 to 10). As a test of the convergence we have done the calculations with 25 bands, which changed the pressure coefficient by about $1 \%$. The summation over the Brillouin zone has been performed by the Monte Carlo method, which appears to be convergent for about 1000 tries.

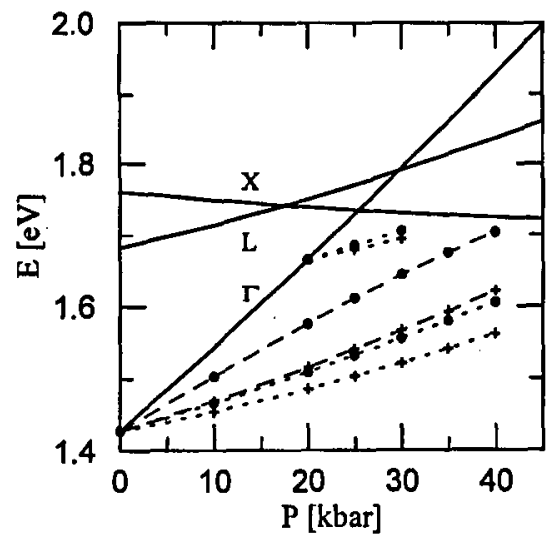

Fig. 1. Calculated energies of the conduction bands and donor states as functions of the hydrostatic pressure. The solid curves labelled by $\Gamma, L$, and $X$ show the results for the conduction band minima. The dashed (dotted) curves correspond to the donor states of the $A_{1}\left(T_{2}\right)$ symmetry and the circles (crosses) - to the donor atom at the anion (cation) site. The energy is measured with respect to the valence-band maximum.

The calculated energies for the conduction band minima and strongly localized donor states are shown in Fig. 1 as functions of pressure $P$. The value of pressure at which the donor level enters the energy gap depends on the unknown short-range potential. We have fitted the parameters $\gamma_{l}$ in the two ways. The first, we have adjusted these parameters in order to get the same crossing point between the donor levels (the four lowest curves in Fig. 1) and the conduction-band minimum at $\Gamma$ for $P=0$. The second, taking on the smaller values of $\gamma_{1}$, we obtain the two upper curves, which are shifted in order to reproduce the more realistic level positions. The pressure coefficients are independent of this shift.

We have considered the following cases: (i) state of the $A_{1}$ symmetry for the group-VI donor at the anion (As) site, (ii) state of the $A_{1}$ symmetry for the 
group-IV donor at the cation (Ga) site, (iii) state of the $T_{2}$ symmetry for the group-VI doror at the anion (As) site, and (iv) state of the $T_{2}$ symmetry for the group-IV donor at the cation (Ga) site. The upward-shifted curves correspond to the states of the $T_{2}$ symmetry. Comparing these results with the experimental data [1-4], we see that the slope of the curve, which corresponds to case (i) is too large. This leads to a suggestion that none of the observed $[2,3]$ group-VI donor states possesses the $A_{1}$ symmetry. The pressure coefficients for cases (ii-iv) are much smaller. The experimental values of pressure coefficients obtained by the various authors differ among themselves by about $2 \mathrm{meV} / \mathrm{kbar}$ [5]. Therefore, we can treat our results as being in agreement with experiment (within the experimental error). This leads to the conclusion that the three possible states of strongly localized donors cannot be excluded from the interpretation of the experimental results [1-4], i.e., states of $A_{1}$ and $T_{2}$ symmetry at the cation (Ga) site, and states of $T_{2}$ symmetry at the anion (As) site. Under the hydrostatic pressure, the shift of the center of gravity of the conduction bands mostly affects the donor pressure coefficients. The different pressure dependence obtained for the considered donor states is due to the different coupling of the Bloch states of the lowest conduction bands with the impurity short-range potential. This coupling depends on the impurity site and the symmetry of the donor states.

The above conclusions should be treated with some caution because they are based on the results of approximate calculations. The band structure is calculated by the empirical pseudopotential method which is not exact enough. The donor states are described by the many-band equation, but with the neglected long-range donor potential. Such approach is more appropriate for isovalent impurities. Summarizing, the present work provides the suggestions for a possible interpretation of the strongly localized donor states in GaAs. The final interpretation should arise as a result of the interplay between the theory and experiment.

\section{References}

[1] X. Liu, L. Samuelson, M.-E. Pistol, M. Gerling, S. Nilsson, Phys. Rev. B 42, 11791 (1990).

[2] J.E. Dmochowski, R.A. Stradling, Jpn. J. Appl. Phys. 32, Suppl. 32-1, 227 (1993).

[3] J.E. Dmochowski, R.A. Stradling, D.J. Dunstan, A.D. Prins, A.R. Adams, K.E. Singer, T. Fujisawa, H. Kukimoto, Mater. Sci. Forum 143-147, 1075 (1994).

[4] J.E. Dmochowski, R.A. Stradling, J. Sly, D.J. Dunstan, A.D. Prins, A.R. Adams, Acta Phys. Pol. A 87, 457 (1994).

[5] S.Y. Ren, J.D. Dow, D.J. Wolford, Phys. Rev. B 25, 7661 (1982).

[6] Z. Wasilewski, R.A. Stradling, Semicond. Sci. Technol. 1, 264 (1986).

[7] W. Trzeciakowski, J. Krupski, Solid Stale Commun. 44, 1491 (1982).

[8] E. Yamaguchi, J. Phys. Soc. Jpn. 56, 2835 (1987).

[9] S. Bednarek, J. Adamowski, in: Proc. 22nd Int. Conf. on the Physics of Semiconductors, Ed. D.J. Lockwood, World Scientific, Singapore 1995, p. 2335.

[10] S. Bednarek, J. Adamowski, Phys. Rev. B 51, 4687 (1995). 